\title{
Using Patient-Level Data to Develop Meaningful Cross-Trial Comparisons of Visual Impairment in Individuals with Diabetic Macular Edema
}

\author{
Sobha Sivaprasad - Stephane A. Regnier · Franck Fajnkuchen • \\ Jonathan Wright · Alan R. Berger · Paul Mitchell · Michael Larsen
}

Received: December 7, 2015 / Published online: March 7, 2016

(c) The Author(s) 2016. This article is published with open access at Springerlink.com

\section{ABSTRACT}

Introduction: The aim of this study was to assess the impact of baseline characteristics on visual outcome of patients with diabetic macular edema and compare the results of clinical trials with different patient populations.

Enhanced content To view enhanced content for this article go to http://www.medengine.com/Redeem/F844 F060559EB5C3.

Electronic supplementary material The online version of this article (doi:10.1007/s12325-016-0310-0) contains supplementary material, which is available to authorized users.

S. Sivaprasad

NIHR Biomedical Research Centre, Moorfields Eye

Hospital, London, UK

S. Sivaprasad

King's College Hospital, London, UK

S. A. Regnier $(\bowtie)$

Novartis Pharma AG, Basel, Switzerland

e-mail: stephane.regnier@novartis.com

F. Fajnkuchen

Centre Ophthalmologique d'Imagerie et de Laser, Paris, France

F. Fajnkuchen

Hôpital Avicenne, Assistance Publique Hôpitaux de Paris, Bobigny, France
Methods: A model was created with patient-level data from the RESPOND/ RESTORE trials to estimate the impact of baseline characteristics on increases in best-corrected visual acuity (BCVA) with anti-vascular endothelial growth factor therapies, measured by letters gained on the Early Treatment Diabetic Retinopathy Study scale from baseline to month 12. Mean BCVA gains with ranibizumab $0.5 \mathrm{mg}$ pro re nata or laser photocoagulation monotherapy were predicted, assuming baseline characteristics equivalent to those in the VIVID-DME/ VISTA-DME trials. These results were compared

J. Wright

Numerus Ltd, Wokingham, UK

A. R. Berger

St Michael's Hospital, Toronto, Canada

P. Mitchell

University of Sydney, Sydney, Australia

M. Larsen

Department of Ophthalmology, Glostrup Hospital, University of Copenhagen, Glostrup, Denmark

M. Larsen

National Eye Clinic, Kennedy Center, Glostrup, Denmark 
with the gain with aflibercept $2.0 \mathrm{mg}$ every 8 weeks in VIVID-DME/VISTA-DME. Sensitivity analyses assessed outcome robustness.

Results: Baseline BCVA and central retinal thickness differed significantly between trials. In unadjusted data, patients in RESPOND/ RESTORE receiving ranibizumab gained an additional 6.6 letters [95\% confidence interval (CI): 4.5-8.7] compared with patients receiving laser monotherapy. After adjusting data to assume baseline characteristics equivalent to VIVID-DME/VISTA-DME, patients receiving ranibizumab were predicted to gain an additional 9.9 letters $\quad(95 \% \quad$ CI: $\quad 7.3-12.4)$ compared with those receiving laser monotherapy. These results were similar (0.1-letter difference in favor of aflibercept; 95\% CI: -2.9 to $3.2 ; P=0.94)$ to the gain in BCVA in patients receiving aflibercept in VIVID-DME/VISTA-DME compared with those receiving laser monotherapy (10.0 letters, 95\% CI: 8.3-11.7).

Conclusion: After adjusting for baseline characteristics, the difference in letters gained between patients receiving ranibizumab versus aflibercept was non-significant across trials, highlighting the importance of adjusting for baseline characteristics in future comparisons.

Funding: Novartis Pharma AG.

Keywords: Aflibercept; Diabetic macular edema; Ophthalmology; Ranibizumab; Vascular endothelial growth factor

\section{INTRODUCTION}

Diabetic macular edema (DME) is a frequent manifestation of diabetic retinopathy that occurs when the retina swells owing to fluid leakage from the macular capillary bed $[1,2]$. DME can lead to visual impairment and, if left untreated, blindness. It is associated with reductions in health-related quality of life and can result in a substantial socioeconomic burden [3, 4].

Until recently, laser photocoagulation monotherapy was the standard of care for DME. Laser monotherapy provides vision stabilization in patients with DME, but offers limited clinically significant improvements in vision [5]. Anti-vascular endothelial growth factor (VEGF) treatments have become available and are now the standard of care for these patients, offering a better prognosis [6]. Ranibizumab, an anti-VEGF-A agent, was the first therapy to receive approval for the treatment of DME [approved by the European Medicines Agency (EMA) in January 2011] [7]. Another anti-VEGF agent, aflibercept, was approved by the EMA for this indication in August 2014 [8].

The approved regimens in the European Union of ranibizumab $0.5 \mathrm{mg}$ pro re nata (PRN) and aflibercept $2.0 \mathrm{mg}$ every 8 weeks have not been compared directly in the same clinical trial. The only study comparing ranibizumab with aflibercept in patients with DME is the DRCR-net Protocol $\mathrm{T}$ trial (ClinicalTrials.gov identifier: NCT01627249) [9]. In Protocol $\mathrm{T}$, patients receiving aflibercept gained, on average, 2.1 letters more than patients receiving ranibizumab $(P=0.03)$. Importantly, that study used a dose of ranibizumab ( $0.3 \mathrm{mg}$ PRN) that is lower than the dose approved outside the United States (0.5 mg PRN). Therefore, the relative effects of ranibizumab $0.5 \mathrm{mg}$ PRN and aflibercept $2.0 \mathrm{mg}$ every 8 weeks remain unclear. In fact, the National Institute for Health and Care Excellence considered that "the results (of Protocol T) could not be considered in its decision making" when appraising ranibizumab $0.5 \mathrm{mg}$ PRN versus aflibercept $2.0 \mathrm{mg}$ every 8 weeks [10]. Therefore, we performed a cross-trial, indirect comparison 
Table 1 Mean gain in BCVA (number of letters) from baseline to month 12, as reported in each of the four published trials RESPOND [11], RESTORE [12], VIVID-DME, and VISTA-DME [13]

\begin{tabular}{|c|c|c|c|}
\hline \multirow[t]{2}{*}{ Trial } & \multirow[t]{2}{*}{ Comparators } & \multicolumn{2}{|c|}{ Mean number of letters gained (SD) } \\
\hline & & $\overline{\text { Anti-VEGF monotherapy }^{a}}$ & Laser monotherapy \\
\hline \multirow[t]{2}{*}{ RESPOND [11] } & Ranibizumab 0.5 mg PRN & $7.5(12.1)$ & $-0.5(13.4)$ \\
\hline & Laser & & \\
\hline \multirow[t]{2}{*}{ RESTORE [12] } & Ranibizumab 0.5 mg PRN & $6.8(8.3)$ & $0.9(11.4)$ \\
\hline & Laser & & \\
\hline \multirow[t]{2}{*}{ VIVID-DME [13] } & Aflibercept $2.0 \mathrm{mg}$ every 8 weeks & $10.7(9.3)$ & $1.2(10.6)$ \\
\hline & Laser & & \\
\hline \multirow[t]{2}{*}{ VISTA-DME [13] } & Aflibercept $2.0 \mathrm{mg}$ every 8 weeks & $10.7(8.2)$ & $0.2(12.5)$ \\
\hline & Laser & & \\
\hline
\end{tabular}

For all reported results, missing data were handled using the last observation carried forward approach. For RESTORE, VIVID-DME, and VISTA-DME, patient data in this table match the published data $[12,13]$. The published data for RESPOND were based on only patients who had measurements at month 12, and showed a mean BCVA gain at month 12 of 8.9 letters for ranibizumab monotherapy and 0.3 letters for laser monotherapy [11]

$B C V A$ best-corrected visual acuity, $P R N$ pro re nata, $S D$ standard deviation, $V E G F$ vascular endothelial growth factor

${ }^{a}$ Either ranibizumab or aflibercept, depending on the trial

incorporating patient-level statistical modeling to compare the visual outcomes of these two treatment regimens. We used clinical trials that compared ranibizumab $0.5 \mathrm{mg}$ PRN (RESPOND [11] and RESTORE [12]; ClinicalTrials.gov identifiers: NCT01135914 and NCT00687804, respectively) or aflibercept $2.0 \mathrm{mg}$ every 8 weeks (VIVID-DME and VISTA-DME; ClinicalTrials.gov identifiers: NCT01331681 and NCT01363440, respectively) [13] with laser monotherapy. Table 1 shows the reported increases in best-corrected visual acuity (BCVA) for each trial, as measured by a gain of letters on the Early Treatment Diabetic Retinopathy Study scale [14]. The description of each trial can be found in the online supplementary material.

\section{METHODS}

A methodology similar to that described here has been used in a previous indirect comparison study [15]; this approach represents a robust procedure for comparing ranibizumab and aflibercept for the treatment of DME. The basic idea behind the model was to leverage the patient-level information in RESPOND/ RESTORE to determine the relationships between baseline characteristics, treatment and BCVA change from baseline to month 12 . Once those relationships had been established, one could predict the outcome of the RESPOND/RESTORE clinical trials if the patients had different baseline characteristics to those in the actual trials.

\section{Base Case Model (Model 1)}

The analysis consisted of four steps.

\section{Step 1: Identification of Confounders}

Published literature was used to identify baseline characteristics considered most likely 
to predict a gain in BCVA at 12 months. Predictors reported to correlate negatively with a gain in BCVA included baseline BCVA [16], central retinal thickness (CRT) [17], and age [16]. Baseline BCVA and CRT differed significantly between RESPOND [11]/RESTORE [12] and VIVID-DME/VISTA-DME [13] (Table 2); these factors were included in the base case model. Age was excluded because mean age did not differ significantly between patients in RESPOND/RESTORE and VIVID-DME/ VISTA-DME (Table 2). In RESTORE, it was shown that the impact on the outcomes of some baseline characteristics, especially CRT, was different between patients receiving ranibizumab and those receiving laser [12]. Therefore, this model also included interaction terms between baseline characteristics and treatment.

\section{Step 2: Regression Model}

The RESPOND and RESTORE patient-level data were appended. A patient-level model analyzed change in BCVA from baseline to month 12 as a function of the baseline values:

$$
\begin{aligned}
Y_{i}= & \beta_{0}+\beta_{1} \text { ranibizumab }_{i}+\beta_{2} \text { ranibizumab } \\
& + \text { laser }_{i}+\beta_{3} \mathrm{BCVA}_{i}+\beta_{4} \mathrm{CRT}_{i}+\beta_{6} \mathrm{BCVA}_{i} \\
& \times \text { ranibizumab }_{i}+\beta_{7} \mathrm{CRT} \times \text { ranibizumab }_{i} \\
& +\beta_{8} \mathrm{BCVA}_{i} \times \text { ranibizumab }+\operatorname{laser}_{i}+\beta_{9} \mathrm{CRT} \\
& \times{\text { ranibizumab }+ \text { laser }_{i}}
\end{aligned}
$$

\begin{tabular}{|c|c|c|c|c|c|c|}
\hline \multirow{2}{*}{ Variables } & \multicolumn{2}{|c|}{ Anti-VEGF monotherapy } & \multirow[t]{2}{*}{$P$ value } & \multicolumn{2}{|c|}{ Laser monotherapy } & \multirow[t]{2}{*}{$P$ value } \\
\hline & $\begin{array}{l}\text { RESPOND/ } \\
\text { RESTORE } \\
191\end{array}$ & $\begin{array}{l}\text { VIVID-DME/ } \\
\text { VISTA-DME } \\
286\end{array}$ & & $\begin{array}{l}\text { RESPOND/ } \\
\text { RESTORE } \\
183\end{array}$ & $\begin{array}{l}\text { VIVID-DME/ } \\
\text { VISTA-DME } \\
286\end{array}$ & \\
\hline BCVA, letters & $64.1(10.3)$ & $59.1(11.0)$ & $<0.001$ & $62.2(10.9)$ & $60.2(10.8)$ & 0.05 \\
\hline $\mathrm{CRT}, \mu \mathrm{m}$ & $435.0(126.0)$ & $497.0(151.0)$ & $<0.001$ & $430.0(128.0)$ & $509.0(153.0)$ & $<0.001$ \\
\hline Age, years & $62.4(9.5)$ & $63.6(8.7)$ & ns & $63.2(9.0)$ & $62.7(8.7)$ & $\mathrm{ns}$ \\
\hline Women, \% & 40 & 42 & ns & 45 & 43 & ns \\
\hline $\mathrm{HbA}_{1 \mathrm{c}}, \%$ & $7.5(1.2)$ & $7.8(1.5)$ & 0.01 & $7.4(1.2)$ & $7.6(1.5)$ & $\mathrm{ns}$ \\
\hline $\begin{array}{l}\text { Patients with } \mathrm{HbA}_{1 \mathrm{c}} \\
\quad>8 \%, \%\end{array}$ & 33 & 35 & ns & 33 & 30 & $\mathrm{~ns}$ \\
\hline $\begin{array}{l}\text { Duration of } \\
\text { diabetes, years }\end{array}$ & $15.7(9.6)$ & $15.9(10.2)$ & ns & $14.4(9.7)$ & $16.0(9.7)$ & $\mathrm{ns}$ \\
\hline
\end{tabular}

Here, $Y_{i}$ represents the change in BCVA from baseline to month 12 [last observation carried

Table 2 Baseline data for RESPOND [11], RESTORE [12], VIVID-DME, and VISTA-DME [13]

Data show mean (standard deviation) unless otherwise indicated

Means were based on weighted averages (weights: patient numbers). Pooled variance for two arms in trial 1 and trial 2 (with $n_{1}$ and $n_{2}$ patients and variances $\operatorname{Var}_{1}$ and $\operatorname{Var}_{2}$, respectively) was calculated using the following formula: $\left(n_{1}-1\right) \times$ $\operatorname{Var}_{1}+\left(n_{2}-1\right) \times \operatorname{Var}_{2} /\left(n_{1}+n_{2}-2\right)$

Statistical analyses: for continuous variables, $P$ values were calculated using a $t$ test (independent samples); for dichotomous variables, $P$ values were calculated using a $\chi^{2}$ test

$B C V A$ best-corrected visual acuity, $C R T$ central retinal thickness, $H b A_{1 c}$ glycated hemoglobin, ns not significant, $V E G F$ vascular endothelial growth factor 
forward (LOCF)] for patient i. Ranibizumab (and ranibizumab + laser $_{i}$ ) is a dichotomous variable equal to 1 if patient $i$ received ranibizumab (or combination therapy) and 0 if patient received laser therapy. $\beta_{0}-\beta_{9}$ are covariate coefficients. Parameter estimates were presented for $\beta_{0}-\beta_{9}$.

The model is interpreted as follows. The predicted mean gain for a patient treated with laser monotherapy and with a mean baseline BCVA of 55 letters and a mean baseline CRT of $500 \mu \mathrm{m}$ is $\beta_{0}+\beta_{3} \times 55+\beta_{4} \times 500$. If the patient receives ranibizumab monotherapy, the mean gain is $\beta_{0}+\beta_{1}+\left(\beta_{3}+\beta_{6}\right) \times 55+\left(\beta_{4}+\beta_{7}\right) \times$ 500 . If the patient receives combination therapy, the mean gain is $\beta_{0}+\beta_{2}+\left(\beta_{3}+\beta_{8}\right) \times$ $55+\left(\beta_{4}+\beta_{9}\right) \times 500$. The mean gain that this patient achieves with ranibizumab monotherapy over laser monotherapy is $\beta_{1}+\beta_{6} \times 55+\beta_{7} \times 500$. The mean gain that this patient achieves with combination therapy over laser monotherapy is $\beta_{2}+\beta_{8} \times 55+\beta_{9} \times 500$. To estimate the relative importance of baseline CRT and BCVA differences between RESPOND/RESTORE and VIVID-DME/VISTA-DME, two models were used: one model with treatment and baseline BCVA as variables and another with treatment and baseline CRT as variables.

\section{Step 3: Predicting Gains in BCVA After} Ranibizumab Treatment Using VIVID-DME/ VISTA-DME Mean Baseline Characteristics

Baseline values from the aflibercept arms in VIVID-DME/VISTA-DME were substituted into the above model to give an estimate of the change in BCVA from baseline to month 12 for ranibizumab monotherapy, assuming that mean patient characteristics in RESPOND were similar to those in VIVID-DME/VISTA-DME. Baseline values from the laser monotherapy arms in VIVID-DME/VISTA-DME were used to predict the mean response for patients receiving this treatment.

\section{Step 4: Indirect Treatment Comparison}

The predicted gains in BCVA with ranibizumab monotherapy and laser monotherapy were used to compare ranibizumab with aflibercept indirectly, using a Bucher method [18].

\section{Base Case Model (Model 1) Validation}

A number of analyses were conducted to assess the robustness of the results, which are presented in the online supplementary material.

\section{Sensitivity Analyses (Models 2-6)}

Sensitivity analyses were performed to ensure that the selection of independent variables was unbiased.

\section{Full Model (Model 2)}

In the first sensitivity analysis, a model was created that included the trial in which the patient participated, baseline variables commonly collected in clinical trials for DME (BCVA, CRT, age, sex, mean $\mathrm{HbA}_{1 \mathrm{c}}$ level, mean duration of diabetes) and the interaction terms between trial, baseline characteristics and treatment.

\section{Stepwise Selection Model (Model 3)}

In the second sensitivity analysis, an automated backward selection process was used to select variables based on their significance level. Selected variables were kept in the model if their $P$ value was $<0.10$. The rationale behind using an automated variable selection process was to remove potential author bias.

Baseline BCVA 24-73 Letters Model (Model 4) Patients were eligible for enrolment in VIVID-DME/VISTA-DME only if their baseline 
BCVA was 24-73 letters. The impact of analyzing only patients in RESPOND/RESTORE with a baseline BCVA of 24-73 letters was assessed.

\section{RESTORE CRT Adjustment Model (Model 5)}

In RESTORE, optical coherence tomography (OCT) was performed at every study visit using Stratus OCT (Carl Zeiss Meditec, Dublin, CA, USA), which is a time-domain OCT (TD-OCT). More recent studies (VIVID-DME/VISTA-DME) [13] used Cirrus OCT (Carl Zeiss Meditec), which is a spectral-domain OCT (SD-OCT) [19]. Because it has been shown that CRT values can be lower when measured with TD-OCT than with SD-OCT $[20,21]$, the RESTORE baseline CRT measurements were converted into SD-CRT values using published data linking Stratus OCT and Cirrus OCT measurements [20]. Specifically, RESTORE baseline CRT was increased by a mean of $39 \mu \mathrm{m}$ [standard deviation (SD): 25] [20].

\section{Baseline BCVA $<69$ Letters Versus $\geq 69$ Letters Model (Model 6)}

The data from model 1 were subdivided to examine the predicted differences in BCVA gain after treatment with ranibizumab in patients with the average baseline characteristics from two Protocol T subgroups (patients with a baseline BCVA of $<69$ letters versus those with a baseline BCVA of $\geq 69$ letters). This predicted result was compared with that published in Protocol T, where patients are similarly subdivided.

\section{Statistical Analyses}

Statistical analyses were based on the full analysis set. Missing values were imputed for the response variable using the LOCF method. SAR and JW conducted the analyses independently. SAR used Stata ${ }^{\circledR}$, version 13.0 (StataCorp LP, College Station, TX, USA), and JW used SAS ${ }^{\circledR}$ version 9.4 (SAS Institute, Cary, NC, USA).

\section{Compliance with Ethics Guidelines}

The RESPOND and RESTORE studies were conducted in accordance with the Declaration of Helsinki and were approved by the ethics committee or institutional review board of each participating center.

\section{RESULTS}

\section{Base Case Model (Model 1)}

For all treatments, model 1 showed that higher baseline BCVA was associated with lower visual acuity gains at 12 months $(P<0.05$; Table 3$)$. The impact of baseline BCVA on BCVA gain did not significantly differ between laser monotherapy, ranibizumab monotherapy, and combination therapy $(P=0.53)$. Higher CRT values at baseline were associated with significantly lower BCVA gains at 12 months for laser monotherapy $(P<0.001$; Table 3$)$. This was not observed for ranibizumab monotherapy or combination therapy $(P=0.12$ and $P=0.49$, respectively).

In the base case model (model 1), the mean predicted gain with ranibizumab monotherapy was 8.7 letters (95\% CI: 7.0-10.5). The mean predicted gain with combination therapy was also 8.7 letters (95\% CI: 6.9-10.6), while there was a mean predicted loss with laser monotherapy of -1.1 letters $(95 \% \mathrm{CI}$ : -3.0 to 0.7$)$. The incremental gain with ranibizumab monotherapy compared with laser monotherapy was 9.9 letters (95\% CI: 7.3-12.4; Table 4; Fig. 1). When the non-significant interaction between baseline BCVA and treatment was removed, the 
Table 3 Coefficient estimations of ranibizumab monotherapy and combination therapy

\begin{tabular}{|c|c|c|c|c|}
\hline Variables & $\begin{array}{l}\text { Base case model } \\
(\operatorname{model} 1)\end{array}$ & $\begin{array}{l}\text { Full model } \\
(\operatorname{model} 2)\end{array}$ & $\begin{array}{l}\text { Stepwise selection } \\
\text { model (model } 3 \text { ) }\end{array}$ & $\begin{array}{l}\text { Baseline BCVA } \\
24-73 \text { letters } \\
\text { model (model 4) }\end{array}$ \\
\hline Baseline BCVA & $-0.180(0.077)^{*}$ & $-0.245(0.083)^{* *}$ & $-0.279(0.049)^{* * *}$ & $-0.105(0.084)$ \\
\hline Baseline CRT & $-0.023(0.006)^{* * *}$ & $-0.028(0.007)^{* * *}$ & $-0.029(0.006)^{* * *}$ & $-0.024(0.006)^{* * *}$ \\
\hline Ranibizumab monotherapy & $-6.743(9.371)$ & $-17.379(16.610)$ & $-8.021(4.018)^{*}$ & $-11.277(9.503)$ \\
\hline Ranibizumab + laser & $2.707(9.469)$ & $15.610(16.967)$ & $-6.472(3.911)$ & $4.790(9.653)$ \\
\hline Ranibizumab $\times$ BCVA at baseline & $-0.010(0.113)$ & $0.016(0.118)$ & - & $0.059(0.123)$ \\
\hline $\begin{array}{l}\text { Ranibizumab }+ \text { laser } \times \\
\text { BCVA at baseline }\end{array}$ & $-0.121(0.116)$ & $-0.148(0.125)$ & - & $-0.169(0.129)$ \\
\hline Ranibizumab $\times$ CRT at baseline & $0.034(0.009)^{* * *}$ & $0.037(0.010)^{* * *}$ & $0.036(0.009)^{* * *}$ & $0.035(0.009)^{* * *}$ \\
\hline $\begin{array}{l}\text { Ranibizumab }+ \text { laser } \times \\
\text { CRT at baseline }\end{array}$ & $0.028(0.009)^{* *}$ & $0.029(0.010)^{* *}$ & $0.032(0.009)^{* * *}$ & $0.029(0.009)^{* *}$ \\
\hline Baseline $\mathrm{HbA}_{1 \mathrm{c}}$ & - & $-0.864(0.714)$ & $-0.725(0.397)$ & - \\
\hline Baseline age & - & $-0.174(0.100)$ & $-0.218(0.054)^{* * *}$ & - \\
\hline Mean duration of diabetes & - & $0.012(0.093)$ & - & - \\
\hline $\operatorname{Sex}(=1$ for women $)$ & - & $-1.664(0.964)$ & - & - \\
\hline Ranibizumab $\times \mathrm{HbA}_{1 \mathrm{c}}$ at baseline & - & $0.865(0.997)$ & - & - \\
\hline $\begin{array}{l}\text { Ranibizumab }+ \text { laser } \times \\
\mathrm{HbA}_{1 \mathrm{c}} \text { at baseline }\end{array}$ & - & $-0.350(1.013)$ & - & - \\
\hline Ranibizumab $\times$ age at baseline & - & $0.034(0.138)$ & - & - \\
\hline $\begin{array}{l}\text { Ranibizumab }+ \text { laser } \times \\
\text { age at baseline }\end{array}$ & - & $-0.153(0.140)$ & - & - \\
\hline $\begin{array}{l}\text { Ranibizumab } \times \text { mean } \\
\text { duration of diabetes }\end{array}$ & - & $-0.060(0.131)$ & - & - \\
\hline $\begin{array}{l}\text { Ranibizumab }+ \text { laser } \times \\
\text { mean duration of diabetes }\end{array}$ & - & $0.055(0.120)$ & - & - \\
\hline Constant & $21.559(6.252)^{* * *}$ & $45.255(11.705)^{* * *}$ & $49.094(7.104)^{* * *}$ & $17.739(6.279)^{* *}$ \\
\hline Observations & 557 & 534 & 534 & 463 \\
\hline$R^{2}$ & 0.143 & 0.185 & 0.173 & 0.157 \\
\hline Adjusted $R^{2}$ & 0.130 & 0.157 & 0.160 & 0.142 \\
\hline
\end{tabular}


Table 3 continued

\begin{tabular}{lllll}
\hline Variables & $\begin{array}{l}\text { Base case model } \\
(\text { model } 1)\end{array}$ & $\begin{array}{l}\text { Full model } \\
(\operatorname{model} 2)\end{array}$ & $\begin{array}{l}\text { Stepwise selection } \\
\text { model (model 3) }\end{array}$ & $\begin{array}{l}\text { Baseline BCVA } \\
24-73 \text { letters } \\
\text { model (model 4) }\end{array}$ \\
\hline BIC & 4233 & 4067 & 4056 & 3451 \\
\hline
\end{tabular}

Standard errors in parentheses

Note: owing to the presence of interaction terms, the coefficients of ranibizumab monotherapy and combination therapy should not be interpreted as treatment effects

$B C V A$ best-corrected visual acuity, $B I C$ Bayesian information criterion, $C R T$ central retinal thickness, $H b A_{1 c}$ glycated hemoglobin

${ }^{*} P<0.05,{ }^{* *} P<0.01,{ }^{* * *} P<0.001$

Table 4 Predicted mean gain in BCVA (number of letters) with ranibizumab over laser monotherapy at month 12 if patients in RESPOND/RESTORE $[11,12]$ had the same baseline characteristics as patients in VIVID-DME/VISTA-DME [13]

\begin{tabular}{lcc}
\hline Model & Mean difference in number of letters & 95\% CI \\
\hline Base case model (model 1) & 9.9 & $7.3-12.4$ \\
Model 1 without interaction between baseline BCVA and treatment & 10.0 & $7.4-12.5$ \\
Model 1 without baseline CRT & 7.7 & $5.3-10.0$ \\
Model 1 without baseline BCVA & 9.3 & $6.8-11.9$ \\
Full model (model 2) & 10.1 & $7.4-12.8$ \\
Stepwise selection model (model 3) & 10.0 & $7.4-12.6$ \\
Baseline BCVA 24-73 letters (model 4) & 9.9 & $7.5-12.4$ \\
RESTORE CRT adjustment model (model 5) & 9.1 & $6.7-11.5$ \\
\hline
\end{tabular}

First assumption: patients receiving ranibizumab have the same mean baseline characteristics as those on aflibercept in VIVID-DME/VISTA-DME [13]. Second assumption: patients receiving laser monotherapy have the same mean baseline characteristics as those on laser monotherapy in VIVID-DME/VISTA-DME [13]

$B C V A$ best-corrected visual acuity, $C I$ confidence interval, $C R T$ central retinal thickness

incremental gain with ranibizumab monotherapy compared with laser monotherapy was 10.0 letters (95\% CI: 7.4-12.5; Table 4).

When the model included baseline BCVA, treatment and interaction between treatment and BCVA, but not baseline CRT, the incremental gain using ranibizumab monotherapy compared with laser monotherapy was 7.7 letters $(95 \%$ CI: 5.3-10.0). When the model included baseline CRT, treatment and interaction terms, but not baseline BCVA, the incremental gain using ranibizumab monotherapy compared with laser monotherapy was 9.3 letters (95\% CI: 6.8-11.9; Table 4).

\section{Sensitivity Analyses}

The estimates of the coefficients for the full model (model 2), the stepwise selection model (model 3), and the baseline BCVA 24-73 letters model (model 4) are shown in Table 3. 


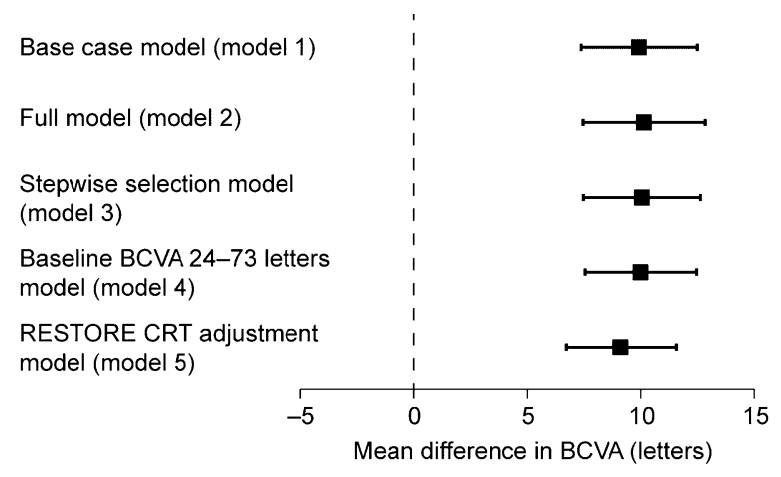

Fig. 1 Ranibizumab $0.5 \mathrm{mg}$ pro re nata versus laser monotherapy. Predicted change in BCVA (95\% confidence interval) from baseline to month 12 if patients in RESPOND [11] and RESTORE [12] had similar baseline characteristics to those in VIVID-DME/ VISTA-DME [13]. Results are shown after adjusting baseline characteristics using the pooled aflibercept $2.0 \mathrm{mg}$ every 8 weeks baseline characteristics from VIVID-DME/ VISTA-DME for ranibizumab predictions and pooled laser monotherapy baseline characteristics from VIVID-DME/VISTA-DME for laser monotherapy predictions. $B C V A$ best-corrected visual acuity, $C R T$ central retinal thickness

Using model 2, the predicted incremental gain in BCVA with ranibizumab over laser monotherapy was 10.1 letters (95\% CI: 7.4-12.8; Table 4). Using model 3, the predicted gain in BCVA was 10.0 letters $(95 \%$ CI: 7.4-12.6). Using model 4, the predicted gain in BCVA was 9.9 letters (95\% CI: 7.5-12.4). Both model 4 and the base case model (model 1) produced similar incremental mean gains over laser monotherapy for ranibizumab (approximately 10 letters). The predicted gain from baseline for ranibizumab was slightly higher in model 4 than in model 1 (9.3 vs. 8.7 letters); however, the loss in vision was slightly less with laser monotherapy in model 4 than in model 1 (-0.6 vs. -1.1 ). Using model 5 , when increasing baseline CRT for patients in RESTORE by a mean of $39 \mu \mathrm{m}$ (SD: $25 \mu \mathrm{m}$ ), the predicted ranibizumab gain over laser monotherapy decreased to 9.1 letters (95\% CI: 6.7-11.5).
In Protocol T, patients with a baseline BCVA of $<69$ letters treated with ranibizumab $0.3 \mathrm{mg}$ gained 5.9 letters more than patients with a baseline BCVA of $\geq 69$ letters (14.2 vs. 8.3 letters gained, a difference of 5.9 letters). Using the same sub-grouping for RESPOND/RESTORE data, model 6 predicts a 3.7-letter difference after ranibizumab $0.5 \mathrm{mg}$ PRN treatment between patients with a baseline BCVA of $\geq 69$ letters and patients with a baseline BCVA of $<69$ letters. Therefore, the differences observed between patients with BCVA of $\geq 69$ letters and patients with a baseline BCVA of $<69$ letters are numerically higher for patients receiving ranibizumab $0.3 \mathrm{mg}$ PRN in Protocol $\mathrm{T}$ than patients receiving $0.5 \mathrm{mg} \mathrm{PRN}$ in RESTORE/RESPOND. Additional research should investigate if this higher difference was due to chance or if ranibizumab $0.5 \mathrm{mg}$ PRN had a bigger effect than ranibizumab $0.3 \mathrm{mg}$ PRN for patients with low baseline BCVA.

\section{Indirect Treatment Comparisons}

Using unadjusted data, the pooled incremental gain in letters for ranibizumab monotherapy over laser monotherapy in RESPOND and RESTORE was 6.6 letters (standard error 1.10) (Table 5). The BCVA mean gain for aflibercept monotherapy over laser monotherapy in VIVID-DME/VISTA-DME [13] was 10.0 letters (95\% CI: 8.3-11.7; Table 5). This compares with the predicted BCVA gain, using adjusted data, in ranibizumab monotherapy compared with laser monotherapy of 9.9 letters (95\% CI: 7.3-12.4; see Table 4). Using those estimates, there was no statistically significant difference in letters gained at 12 months between ranibizumab and aflibercept (0.1-letter difference; $95 \% \mathrm{CI}$ : -2.9 to $3.2 ; \quad P=0.94$; Fig. 2). There was also no statistically significant difference between the gain with 
Table 5 Incremental number of letters gained with aflibercept treatment compared with laser monotherapy in patients from RESPOND/RESTORE [11, 12] or from VIVID-DME/VISTA-DME [13]

\begin{tabular}{|c|c|c|}
\hline \multirow[t]{2}{*}{ Treatment } & \multicolumn{2}{|c|}{ Incremental number of letters gained (SE) } \\
\hline & $\overline{\text { RESPOND }}$ & RESTORE \\
\hline Ranibizumab & $7.5(1.39)$ & $6.8(0.77)$ \\
\hline Laser monotherapy & $-0.5(1.58)$ & $0.9(1.09)$ \\
\hline Ranibizumab incremental gain vs. laser monotherapy & $8.0(1.93)$ & $5.9(1.33)$ \\
\hline $\begin{array}{l}\text { Pooled ranibizumab incremental gain vs. laser monotherapy } \\
\text { (pooled RESPOND/RESTORE) })^{a}\end{array}$ & \multicolumn{2}{|c|}{$6.6(1.10)$} \\
\hline Treatment & VIVID-DME & VISTA-DME \\
\hline Aflibercept & $10.7(0.80)$ & $10.7(0.67)$ \\
\hline Laser monotherapy & $1.2(0.92)$ & $0.2(1.01)$ \\
\hline Aflibercept incremental gain vs. laser monotherapy & $9.5(1.22)$ & $10.5(1.21)$ \\
\hline $\begin{array}{l}\text { Pooled aflibercept incremental gain vs. laser monotherapy } \\
\text { (pooled VIVID-DME/VISTA-DME) }^{\mathrm{a}}\end{array}$ & \multicolumn{2}{|c|}{$10.0(0.86)$} \\
\hline
\end{tabular}

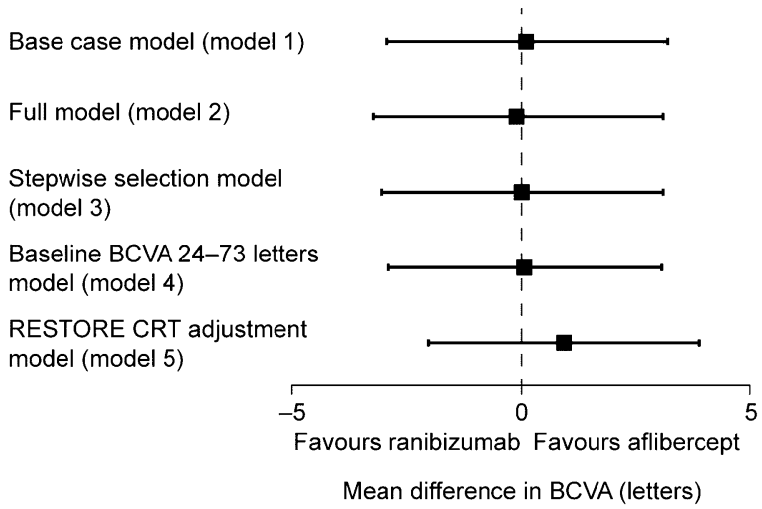

Fig. 2 Indirect treatment comparison (95\% confidence interval) between ranibizumab $0.5 \mathrm{mg}$ pro re nata and aflibercept $2.0 \mathrm{mg}$ every 8 weeks. Ranibizumab-predicted values after adjustment for baseline characteristics were used in the analysis. $B C V A$ best-corrected visual acuity, $C R T$ central retinal thickness

ranibizumab and that with aflibercept in any of the other models. Model 5 (RESTORE CRT adjustment model) showed the largest difference between aflibercept and ranibizumab, although this was non-significant $(P=0.54)$.

\section{DISCUSSION}

Our model predicted that, if patients in RESPOND/RESTORE had similar BCVA and CRT as in VIVID/VISTA, the incremental letter gains of ranibizumab $0.5 \mathrm{mg}$ over laser would have been greater than those observed in RESTORE/RESPOND. More specifically, the ranibizumab incremental response to laser monotherapy would have been greater than the unadjusted data by a margin of 3.3 letters. The main driver of this change in response was the difference in baseline CRT between RESPOND/RESTORE and VIVID-DME/ VISTA-DME. Baseline CRT also had a higher impact on outcomes for laser monotherapy than for ranibizumab. Specifically, if two patients undergoing laser monotherapy had a difference of $100 \mu \mathrm{m}$ in baseline CRT, BCVA gain would be, on average, 2.3 letters less for the patient with the higher baseline CRT. A similar relationship between baseline CRT and laser 
monotherapy was observed in VIVID-DME/ VISTA-DME. Patients receiving laser monotherapy with a baseline CRT $\geq 400 \mu \mathrm{m}$ gained 3 more letters than patients with a baseline CRT $<400 \mu \mathrm{m}$ [22]. Protocol $\mathrm{T}$ also found a substantial impact of CRT at baseline $(P=0.01)$ [9]. For instance, patients receiving aflibercept with a baseline CRT $\geq 400 \mu \mathrm{m}$ gained 5.3 more letters than patients with a baseline CRT $<400 \mu \mathrm{m}$ and patients receiving ranibizumab $0.3 \mathrm{mg}$ with a baseline CRT $\geq 400 \mu \mathrm{m}$ gained 2.3 more letters than patients with a baseline CRT $<400 \mu \mathrm{m}$.

The incremental gains in BCVA using ranibizumab over laser monotherapy ranged from 9.9 to 10.0 letters across models 1-4, which used data from RESPOND and RESTORE, the narrow range showing the robustness of the base case model results. Furthermore, additional variables used across models were not statistically different between the trials of interest. Across all models, model 5 had the largest impact with a predictive gain of 9.1 letters, while model 4 had the smallest impact, producing a predictive gain of 9.9 letters. Nevertheless, the value from model 5 remained well above the unadjusted gain of 6.6 letters.

The absolute letter gains at month 12 as well as the differences in letter gain between sub-groups (patients with a baseline BCVA of $<69$ letters versus those with a baseline BCVA of $\geq 69$ letters) are less pronounced in our analyses than in Protocol T.

The current analysis underlines the importance of adjusting for key confounders when comparing clinical trial data. If the unadjusted results from RESPOND/RESTORE and VIVID-DME/VISTA-DME are compared in a naïve indirect treatment comparison, aflibercept would appear statistically superior to ranibizumab $0.5 \mathrm{mg}$ PRN. However, this analysis demonstrates that this statistical difference is driven by differences in patient characteristics between trials.

Some remaining unobserved heterogeneity may exist between studies even after adjusting for key baseline characteristics. More details on the study limitations are given in the online supplementary material.

Two network meta-analyses in DME have been published [23, 24]. Additional research could include using the adjusted clinical trials outcomes of the analysis to update the network meta-analyses.

\section{ACKNOWLEDGMENTS}

This study was funded by Novartis Pharma AG, Basel, Switzerland. The article processing charges and open access fee for this publication were funded by Novartis Pharma AG. Medical writing support was provided by Dr. Noëlle L. O'Regan of PharmaGenesis London and was funded by Novartis Pharma AG. All named authors meet the International Committee of Medical Journal Editors (ICMJE) criteria for authorship for this manuscript, take responsibility for the integrity of the work as a whole, and have given final approval for the version to be published.

Disclosures. Sobha Sivaprasad has received financial support from Allergan, Bayer and Novartis. She has received travel grants and honoraria from Allergan, Bayer, Novartis and Roche for advisory board meetings. She also acknowledges support from NIHR Moorfields Biomedical Research Centre in conducting this study. Stephane A. Regnier is an employee of Novartis Pharma AG, Basel, Switzerland. Franck Fajnkuchen is a consultant for Allergan, Bayer and Novartis. Jonathan Wright is an employee 
of Numerus Ltd, Wokingham, UK. Numerus Ltd was paid by Novartis to conduct analyses for this manuscript. Alan R. Berger has received honoraria from Alcon, Bayer, Bausch + Lomb and Novartis. His institution has received grants and research funding from Alcon, Bayer and Novartis. He has served on advisory boards for Alcon, Bayer and Novartis. Paul Mitchell has been a consultant for Abbott, Allergan, Bayer, Novartis and Roche, and has received honoraria and travel expenses from these companies. Michael Larsen is a consultant for Allergan, AstraZeneca, GlaxoSmithKline, Novartis, Novo Nordisk and Roche, and has received financial support from Allergan, GlaxoSmithKline, Novartis and Roche.

Compliance with Ethics Guidelines. The RESPOND and RESTORE studies were conducted in accordance with the Declaration of Helsinki and were approved by the ethics committee or institutional review board of each participating center.

Open Access. This article is distributed under the terms of the Creative Commons Attribution-NonCommercial 4.0 International License (http://creativecommons.org/licenses/ by-nc/4.0/), which permits any noncommercial use, distribution, and reproduction in any medium, provided you give appropriate credit to the original author(s) and the source, provide a link to the Creative Commons license, and indicate if changes were made.

\section{REFERENCES}

1. Cheung $\mathrm{N}$, et al. Diabetic retinopathy. Lancet. 2010;376:124-36.

2. Ciulla TA, et al. Diabetic retinopathy and diabetic macular edema: pathophysiology, screening, and novel therapies. Diabetes Care. 2003;26:2653-64.
3. Davidov E, et al. Diabetic retinopathy and health-related quality of life. Graefes Arch Clin Exp Ophthalmol. 2009;247:267-72.

4. Matza LS, et al. The longitudinal link between visual acuity and health-related quality of life in patients with diabetic retinopathy. Health Qual Life Outcomes. 2008;6:95.

5. Diabetic Retinopathy Guidelines. https://www. rcophth.ac.uk/wp-content/uploads/2014/12/2013SCI-301-FINAL-DR-GUIDELINES-DEC-2012-updatedJuly-2013.pdf; 2012. Accessed 19 March 2015.

6. Bandello F, et al. New approaches for the treatment of diabetic macular oedema: recommendations by an expert panel. Eye (Lond). 2012;26:485-93.

7. Lucentis (ranibizumab) summary of product characteristics. http://www.ema.europa.eu/docs/en GB/document_library/EPAR_-_Product_Information/ human/000715/WC500043546.pdf. Accessed 19 March 2015.

8. Eylea (aflibercept) summary of product characteristics. http://www.ema.europa.eu/docs/en GB/document_library/EPAR_-_Product_Information/ human/002392/WC500135815.pdf. Accessed 19 March 2015.

9. DRCR-Network, et al. Aflibercept, bevacizumab, or ranibizumab for diabetic macular edema. N Engl J Med. 2015;372:1193-203.

10. National Institute for Health and Care Excellence. Final appraisal determination: aflibercept for treating diabetic macular oedema. https://www.nice.org.uk/ guidance/gid-tag472/documents/macular-oedemadiabetic-aflibercept-final-appraisal-determinationdocument2; 2015. Accessed 03 July 2015.

11. Berger A, et al. Efficacy/safety of ranibizumab monotherapy or with laser versus laser monotherapy in DME. Can J Ophthalmol. 2015;50:209-16.

12. Mitchell $\mathrm{P}$, et al. RESTORE: ranibizumab monotherapy or combined with laser versus laser monotherapy for diabetic macular edema. Ophthalmology. 2011;118:615-25.

13. Korobelnik JF, et al. Intravitreal aflibercept for diabetic macular edema. Ophthalmology. 2014;121:2247-54.

14. Jansen JP, Naci H. Is network meta-analysis as valid as standard pairwise meta-analysis? It all depends on the distribution of effect modifiers. BMC Med. 2013;11:159.

15. Nixon R, et al. No evidence of disease activity: indirect comparisons of oral therapies for the 
treatment of relapsing-remitting multiple sclerosis. Adv Ther. 2014;31:1134-54.

16. Bressler SB, et al. Factors associated with changes in visual acuity and central subfield thickness at 1 year after treatment for diabetic macular edema with ranibizumab. Arch Ophthalmol. 2012;130:1153-61.

17. Rayess $\mathrm{N}$, et al. Baseline choroidal thickness as a predictor for response to anti-vascular endothelial growth factor therapy in diabetic macular edema. Am J Ophthalmol. 2015;159:85-91.

18. Bucher HC, et al. The results of direct and indirect treatment comparisons in meta-analysis of randomized controlled trials. J Clin Epidemiol. 1997;50:683-91.

19. Do DV. Using aflibercept to treat diabetic macular edema. Retina Today 2014;Apr:55-6.

20. Bressler SB, et al. Reproducibility of spectral-domain optical coherence tomography retinal thickness measurements and conversion to equivalent time-domain metrics in diabetic macular edema. JAMA Ophthalmol. 2014;132:1113-22.
21. Mathew R, et al. Agreement between time-domain and spectral-domain optical coherence tomography in the assessment of macular thickness in patients with idiopathic macular telangiectasia type 2 . Ophthalmologica. 2013;230:144-50.

22. Ogura $Y$, et al. Effect of baseline central retinal thickness (CRT) and best corrected visual acuity (BCVA) on treatment outcomes with intravitreal aflibercept injection (IAI) or macular laser photocoagulation in diabetic macular edema (DME). Association for Research in Vision and Ophthalmology 2015; Poster Board Number: A0181.

23. Régnier S, et al. Efficacy of anti-VEGF and laser photocoagulation in the treatment of visual impairment due to diabetic macular edema: a systematic review and network meta-analysis. PLoS One 2014;9(7):e102309.

24. Korobelnik JF, et al. Systematic review and mixed treatment comparison of intravitreal aflibercept with other therapies for diabetic macular edema (DME). BMC Ophthalmol. 2015;15(15):52. 